\section{Reprodutibilidade do questionário de avaliação de atividade física para crianças aplicado no Estudo Saúdes - Vitória}

\section{The reproducibility of the Saúdes - Vitória Study's physical activity assessment questionnaire for children}

\begin{abstract}
Objectives: to evaluate the reproducibility of a physical activity assessment questionnaire for children aged between 7 and 10 years among participants of the Saúdes Vitória Study in Espírito Santo State, Brazil.

Methods: a cross-sectional study was carried out in a random sample of 91 children enrolled in public and private sector schools in the municipality. A structured questionnaire with 13 questions was sent to the home of selected children, to be filled out twice, with an interval of 15 days. To evaluate the reproducibility the Kappa agreement test was used for the qualitative variables, and Pearson's correlation or Spearman's $r$ for the quantitative ones, followed by a scatter analysis.

Results: the Kappa agreement indices were significant for all qualitative variables and varied from moderate to high ( $k=0.72-0.98)$. Likewise, the correlation coefficients ranged from moderate to high $(r=0.51-1.00)$, except for time spent playing video games and duration of second sport, which showed no significant correlation.

Conclusions: Saúdes questionnaire enabled data on physical and sedentary activities to be collected with a good level of reproducibility, although there are limitations in the population under study regarding the quantification of the time spent on less frequent activities, such as playing video games or practicing a second sport.
\end{abstract}

Key words Students, Motor activity, Reproducibility of results, Questionnaires
Karina Checon 1

Vivian Moro Fonseca 2

Carolina Perim de Faria 3

Luciana Carletti 4

Maria del Carmen Bisi Molina 5

1,2 Universidade Federal do Espírito Santo. Vitória, ES, Brasil. ${ }^{3}$ Escola Nacional de Saúde Pública. Fundação Oswaldo Cruz. Rio de Janeiro, RJ, Brasil.

4 Programa de Pós-graduação em Educação Física. Universidade Federal do Espírito Santo. Vitória, ES, Brasil.

5 Programa de Pós-graduação em Saúde Coletiva. Centro de Ciências da Saúde. Universidade Federal do Espírito Santo. Av. Marechal Campos, 1468. Maruípe. Vitória, ES, Brasil. CEP: 29.040-090. E-mail:mdmolina@uol.com.br

\title{
Resumo
}

Objetivos: avaliar a reprodutibilidade de um questionário de atividade física (AF) para crianças de 7 a 10 anos em participantes do estudo Saúdes Vitória, Espirito Santo, Brasil.

Métodos: estudo de corte transversal realizado em uma amostra aleatória de 91 crianças matriculadas em escolas da rede pública e privada do municipio. Um questionário estruturado com 13 perguntas foi enviado ao domicilio do escolar para preenchimento em duas ocasiões, com intervalo de 15 dias entre elas. $O$ teste de concordância de Kappa foi utilizado na avaliação da reprodutibilidade das variáveis qualitativas, e o teste de correlação de Pearson ou de Spearman na avaliação das variáveis quantitativas, seguido da análise de dispersão.

Resultados: os indices de concordância Kappa variaram de moderados a altos e foram estatisticamente significantes para todas as variáveis qualitativas, e $(k=0,72$ a 0,98). Da mesma forma, os valores dos coeficientes de correlação para as respostas numéricas variaram de moderado a alto $(r=0,51$ a $1,00)$, exceto para o tempo de uso de vídeo game e duração da prática de segunda modalidade esportiva.

Conclusões: o questionário Saúdes permitiu coletar dados sobre AF com boa reprodutibilidade, porém apresentou limitações na quantificação do tempo de atividades de menor frequência diária, como o tempo de uso do vídeo game e tempo de uma segunda prática esportiva.

Palavras-chave Estudantes, Atividade motora, Reprodutibilidade dos resultados, Questionários 


\section{Introdução}

A atividade física (AF) é um importante fator na promoção de saúde, estando sua prática relacionada à efeitos benéficos aos sistemas cardiovascular, metabólico e locomotor. ${ }^{1,2}$

Estudos mostram que crianças e adolescentes que se mantêm ativos fisicamente apresentam menor chance de se tornarem adultos sedentários. ${ }^{3-5}$ Desta forma, o monitoramento dos níveis de prática habitual de AF na população infantil tem-se tornado tema de interesse entre especialistas da área, 6,7 embora haja poucos estudos nacionais abrangendo esta população. ${ }^{8}$

Estudos epidemiológicos que avaliam a prática de atividades físicas tem-se mostrado importantes, especialmente aqueles que visam identificar fatores de risco ou de proteção para doenças crônicas. A identificação do padrão de atividade física de crianças e jovens requer a consideração de aspectos relacionados à duração, intensidade, frequência, tipo e, local da prática da atividade, além de fatores como o clima, para o melhor entendimento da dinâmica deste comportamento. 9

Diferentes métodos têm sido empregados na avaliação dos níveis de atividade física, dentre eles, o monitoramento da frequência cardíaca por equipamentos, uso de sensores de movimento, método da água duplamente marcada, observação direta, e métodos de auto-relato (entrevistas, recordatórios, questionários e diários).10 Os questionários e as entrevistas são os mais utilizados em estudos epidemiológicos pelo fato de apresentarem boa concordância com os instrumentos de observação direta, facilidade de aplicação e baixo custo.11 Ressalta-se, ainda, o fato de serem capazes de se obter dados sobre duração, frequência, intensidade e tipo da atividade física.

Contudo, devido a menor capacidade cognitiva das crianças muito jovens, 12 a aplicação de questionários tem sido restrita nessa população, levando alguns pesquisadores a utilizarem informações fornecidas pelos pais ou pessoas próximas às crianças como referência. ${ }^{6}$

Embora o questionário "Dia típico de atividade física e alimentação" (DAFA) seja bastante empregado em estudos no âmbito nacional, 6,13 esse instrumento apenas avalia os aspectos qualitativos da atividade física, fato que tem limitado a quantificação do nível de atividade física, como o tempo total despendido com atividades físicas por semana. ${ }^{8}$ Sendo assim, um questionário foi desenvolvido com objetivo de determinar o nível de atividade física de crianças com idade entre sete e dez anos, que partici- param da pesquisa Saúdes-Vitória, que foi realizada na cidade de Vitória, Espírito Santo. A pesquisa tratou-se de um estudo seccional em uma amostra de 1282 escolares, que avaliou o estado nutricional e de saúde de escolares no primeiro ciclo do ensino fundamental. O presente trabalho testou a reprodutibilidade do referido questionário, em uma subamostra da população que participou do referido estudo.

\section{Métodos}

O instrumento foi aplicado em uma amostra de 91 escolares que participaram do Estudo SaúdesVitória, matriculados no ensino fundamental de escolas públicas e privadas da cidade de Vitória no Espírito Santo, em 2007. A seleção das escolas públicas e particular foi feita por meio de um sorteio entre as escolas participantes do Projeto SaúdesVitória.

A coleta de dados foi realizada entre outubro e dezembro de 2007. As informações sobre os hábitos de atividades físicas, padrões comportamentais e estilo de vida das crianças foram obtidas por meio do questionário, o qual foi enviado diretamente às mães ou responsáveis das crianças selecionadas. O preenchimento do instrumento foi preferencialmente feito pelas mães ou cuidadores diretos.

A participação das crianças na pesquisa esteve na dependência da autorização da mãe ou responsável por meio da assinatura do termo de consentimento livre e esclarecido. Este estudo foi aprovado pelo Comitê de Ética em Pesquisa da Universidade Federal do Espírito Santo (UFES), sendo registrado sob o número CEP/UFES 089/06.

As informações sobre atividade física foram obtidas por meio da aplicação de perguntas relativas à forma de locomoção da criança do domicílio à escola, o tempo despendido para chegar à escola, quando tal deslocamento não era realizado em veículos, prática de um ou mais esportes fora da escola e o tempo despendido em cada um deles (denominados Esporte 1, 2 e 3), tempo diário despendido em atividades de lazer ativo e em lazer sedentário (uso de equipamentos eletrônicos: televisão, vídeo game e computador).

O questionário foi enviado em dois momentos distintos, com intervalo de quinze dias entre ambos, para verificar a estabilidade das respostas. Uma carta explicando a finalidade da pesquisa e enfatizando a necessidade do preenchimento dos dois questionários por uma única pessoa foi enviada aos pais ou responsáveis.

O teste de concordância de Kappa (coeficiente 
de concordância para escalas nominais - k) foi utilizado para avaliar o grau de concordância entre as respostas de natureza qualitativa (variáveis dicotômicas), enquanto os teste de correlação de Pearson ou de Spearman foram utilizados para avaliar a concordância entre os valores obtidos pelas respostas de natureza numérica (variáveis quantitativas). Os índices de concordância e correlação foram classificados em: $>0,75$ - excelente; $\geq 0,4$ e $\leq 0,75$ - médio a bom; e $<0,40$ - baixo. ${ }^{14}$ A dispersão dos dados quantitativos e o nível de concordância entre as duas medidas foi apresentada e comparada utilizando-se da técnica de Bland e Altman. ${ }^{10}$ Para todas as análises foi adotado um nível de significância de 5\%.

\section{Resultados}

Um total de 130 questionários foram enviados aos domicílios dos escolares sorteados. Destes, 80 foram remetidos às residências dos estudantes de escolas públicas, tendo 50 sido aplicados nos dois momentos (testes e re-testes). Dos 50 questionários remetidos às residências dos alunos da escola particular, 42 foram respondidos nos momentos do teste e re-teste. Assim, um total de 91 questionários foram incluídos no estudo, uma vez que uma criança estava fora da faixa etária.

Conforme apresentado na Tabela 1, 54,0\% crianças são do sexo feminino e $54,0 \%$ estudavam em escolas públicas. A maioria (64,0\%) tinha entre nove e dez anos de idade, $41,8 \%$ participantes foram classificados, pelos seus pais, como brancos e $78,0 \%$ moravam com o pai e com a mãe.

Com relação às mães ou cuidadoras das crianças, a maioria tinha idade entre 30 e 39 anos e nível fundamental de escolaridade. Quanto à situação ocupacional das mães, com atividades laborativas fora do domicílio, observou-se que $64,8 \%$ estavam empregadas e/ou eram profissionais liberais.

A Tabela 2 mostra os resultados encontrados no teste/re-teste para a análise de concordância Kappa, e coeficiente de correlação de Pearson ou Spearman. As perguntas relativas às atividades de rotina dos filhos, tais como, meio de locomoção de casa para a escola, assistir televisão, jogar videogame, usar computador, participação em atividades desportivas e o tipo de esporte praticado obtiveram concordância

Tabela 1

Caracterização da sub-amostra de escolares do Estudo Saúdes. Vitória, ES, 2007.

\begin{tabular}{|c|c|c|c|c|}
\hline \multirow{2}{*}{ Variável } & \multicolumn{2}{|c|}{ Sub-amostra estudada } & \multicolumn{2}{|c|}{ Amostra Projeto Saúdes } \\
\hline & $\mathrm{n}$ & $\%$ & $\mathrm{n}$ & $\%$ \\
\hline \multicolumn{5}{|l|}{ Sexo } \\
\hline Masculino & 42 & 46,0 & 538 & 42,0 \\
\hline Feminino & 49 & 54,0 & 744 & 58,0 \\
\hline \multicolumn{5}{|l|}{ Tipo de escola } \\
\hline Pública & 49 & 54,0 & 1061 & 82,8 \\
\hline Privada & 42 & 46,0 & 221 & 17,2 \\
\hline \multicolumn{5}{|l|}{ Faixa etária (anos) } \\
\hline 7 & 14 & 16,0 & 252 & 19,6 \\
\hline 8 & 18 & 20,0 & 359 & 28,0 \\
\hline 9 & 31 & 34,0 & 360 & 28,1 \\
\hline 10 & 27 & 30,0 & 311 & 24,2 \\
\hline \multicolumn{5}{|l|}{ Idade da mãe (anos) } \\
\hline$<30$ & 13 & 14,0 & 193 & 15,0 \\
\hline $30-39$ & 47 & 52,0 & 704 & 55,0 \\
\hline$\geq 40$ & 31 & 34,0 & 385 & 30,0 \\
\hline \multicolumn{5}{|l|}{ Escolaridade da mãe (anos) } \\
\hline Fundamental completo ou menos & 39 & 42,8 & 580 & 45,2 \\
\hline Médio completo & 29 & 31,9 & 456 & 35,6 \\
\hline Superior & 23 & 25,3 & 246 & 19,2 \\
\hline \multicolumn{5}{|l|}{ Situação empregatícia da mãe } \\
\hline Empregada/Profissional liberal & 59 & 64,8 & 848 & 67,6 \\
\hline Desempregada/Dona de casa & 32 & 35,2 & 406 & 32,4 \\
\hline
\end{tabular}


moderada a alta $(\mathrm{k}=0,72$ a 0,98$)$. A questão relacionada à terceira modalidade de esporte não foi incluída porque apenas uma mãe respondeu.

Para as questões de natureza quantitativa (Tabela 2), a maioria obteve coeficientes de correlação moderados a altos $(\mathrm{r}=0,51$ a 1,00$)$ e $p<0,05$. No entanto, a análise do tempo de uso de vídeo game ( $\mathrm{r}=$ $0,25, p=0,33$ ) e duração do segundo esporte, quando referido, $(\mathrm{r}=-0,01, p=0,97)$, não apresentaram correlação estatisticamente significante. A pergunta referente ao tempo de deslocamento do ponto de ônibus à escola apresentou correlação perfeita $(\mathrm{r}=1,00)$, contudo apenas três mães ou cuidadoras apresentaram respostas para este item.
Os resultados da análise da reprodutibilidade das questões relativas ao tempo de atividade física diária informado pelos entrevistados é apresentada na Figura 1. Apenas o tempo de deslocamento à escola, de prática semanal de esportes e tempo de atividades recreativas foram incluídos na apresentação, uma vez que tiveram correlação estatisticamente significante.

O tempo diário de deslocamento à escola entre as duas aplicações se concentrou entre 6 a 13 min, com uma dispersão regular, considerando que cerca de $50 \%$ dos resultados se concentraram na média da diferença, ou muito próximo da mesma (Figura 1A).

Tabela 2

Relação entre as respostas do teste e re-teste do questionário aplicado às mães ou cuidadoras de crianças de sete a dez anos de idade. Vitória, ES, 2007.

\begin{tabular}{|c|c|c|c|}
\hline Pergunta / Variável & $\mathbf{N}$ & Coeficiente & $p$ \\
\hline 1 - Como o seu filho (a) vai para a escola? & 89 & 0,92 & $<0,001$ \\
\hline $\begin{array}{l}2 \text { - Se seu filho (a) vai a pé ou de bicicleta, quanto tempo ele (a) gasta para } \\
\text { chegar à escola? (em minutos) }\end{array}$ & 50 & 0,77 & $<0,001$ \\
\hline $\begin{array}{l}3 \text { - Se seu filho (a) vai de ônibus, quanto tempo ele (a) anda para chegar ao } \\
\text { ponto de ônibus? (em minutos) }\end{array}$ & 3 & 0,87 & $<0,001$ \\
\hline $\begin{array}{l}4 \text { - Se seu filho (a) vai de ônibus, quanto tempo ele(a) precisa andar do ponto de } \\
\text { ônibus à escola? (em minutos) }\end{array}$ & 3 & 1,00 & $<0,001$ \\
\hline 5 - Seu filho (a) assiste à televisão todos os dias? & 91 & 0,98 & $<0,001$ \\
\hline $\begin{array}{l}6 \text { - Se respondeu sim a questão anterior, diga quanto tempo ele (a) assiste } \\
\text { televisão por dia. (em minutos) }\end{array}$ & 84 & 0,51 & $<0,001$ \\
\hline 7 - Seu filho (a) joga videogame? & 91 & 0,78 & $<0,001$ \\
\hline $\begin{array}{l}8 \text { - Se respondeu sim a questão anterior, diga quanto tempo ele (a) joga } \\
\text { videogame por dia. (em minutos) }\end{array}$ & 31 & 0,25 & 0,326 \\
\hline 9 - Seu filho(a) usa computador? & 90 & 0,79 & $<0,001$ \\
\hline $\begin{array}{l}10 \text { - Se respondeu sim a questão anterior, diga quanto tempo ele(a) usa o } \\
\text { computador por dia. (em minutos) }\end{array}$ & 59 & 0,64 & $<0,001$ \\
\hline $\begin{array}{l}11 \text { - Seu filho participa de alguma escolinha, time ou treinamento desportivo? } \\
\text { (desde que haja treinador/professor). }\end{array}$ & 91 & 0,74 & $<0,001$ \\
\hline Esporte 1 & 17 & 0,87 & $<0,001$ \\
\hline Esporte 2 & 11 & 0,72 & $<0,001$ \\
\hline Esporte 3* & 1 & 1 & $<0,001$ \\
\hline $\begin{array}{l}12 \text { - Se respondeu sim à questão 12, informe qual (is) esporte(s) e a duração (em } \\
\text { minutos) da atividade por semana }\end{array}$ & & & \\
\hline Duração total por semana esporte 1 & 17 & 0,60 & 0,01 \\
\hline Duração total por semana esporte 2 & 11 & $-0,01$ & 0,97 \\
\hline Duração total por semana esporte 3 & 1 & * & * \\
\hline $\begin{array}{l}13 \text { - Por quantas horas diárias seu filho (a) brinca ativamente (joga futebol, corre, } \\
\text { dança, nada, anda de bicicleta, brinca de pique etc)? }\end{array}$ & 32 & 0,80 & $<0,001$ \\
\hline
\end{tabular}

Coeficiente de correlação Kappa (k), para variáveis qualitativas e coeficientes de correlação de Pearson ou Spearman, para variáveis quantitativas. *Feita a pergunta, porém somente uma mãe mencionou que seu filho faz três esportes diferentes. Os números relativos às perguntas do questionário original formam mantidos na tabela para fornecer informações sobre a organização do questionário completo. 
Diferenças das informações sobre as médias de tempo gasto em deslocamento (A), esportes (B) e brincadeiras ativas (C) obtidas pela aplicação das duas réplicas do questionário.
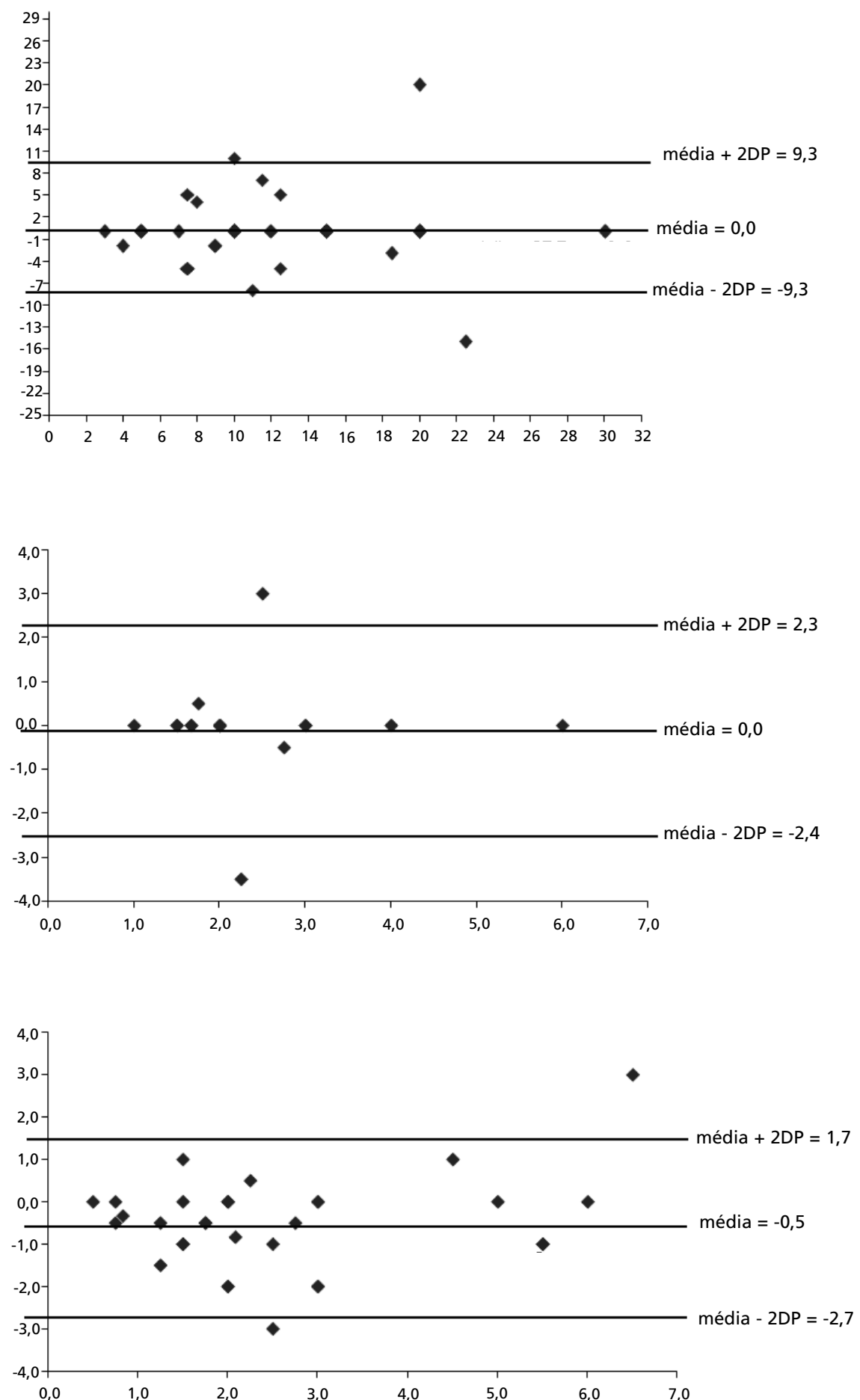

c

Eixo das ordenadas: diferenças entre as médias do tempo diário de atividade física referido na primeira e segunda aplicação do questionário. Eixo das abscissas: média do tempo de atividade física entre as réplicas.

(A) tempo diário de deslocamento à escola em minutos; (B) tempo semanal de prática de esportes em horas; (C) tempo diário de brincadeiras em horas. 
As informações sobre o tempo despendido assistindo televisão apresentou grande dispersão em torno da média. A média das diferenças entre os dois questionários foi de 0,4 horas/dia, e muitos valores se distanciaram desta referência. Quanto às informações sobre o tempo de uso diário de computador, a média das diferenças entre as réplicas foi de 0,1 hora/dia, com desvio padrão de 0,5 horas/dia (dados não apresentados em figura).

A prática semanal do esporte 1 apresentou bons limites de concordância entre as réplicas (Figura 1B), com muitas diferenças nos valores entre indivíduos.

Para o tempo diário de brincadeiras observa-se uma dispersão regular com a média das diferenças entre as duas aplicações situando-se em - $-0,5$ horas/dia. É possível ainda visualizar que a maior parte dos resultados se concentra em até três horas de brincadeiras diárias (Figura 1C).

\section{Discussão}

Em virtude da facilidade de aplicação e de seu baixo custo, a avaliação da reprodutibilidade de questionários que avaliam a atividade física de crianças em estudos epidemiológicos torna-se importante. Porém, há dificuldades para se trabalhar com estes instrumentos, quando respondidos pelos participantes do estudo, pois caso não haja adequado entendimento das questões, pode-se incorrer em erros, diminuindo a reprodutibilidade desta ferramenta de coleta de dados. 14,15

Nesse estudo, o entendimento das questões por parte das mães/cuidadores das crianças pode ter sido um dos problemas encontrados para a baixa reprodutibilidade das perguntas relacionadas ao tempo de execução de atividades físicas ou sedentárias. Outra possibilidade é a falta de rotina pré-estabelecida para crianças, que resultaria em dificuldades para se calcular o tempo despendido em atividades diárias.

Esta limitação foi previamente documentada no estudo realizado por Welk et al.,12 onde os autores destacam a dificuldade de se mensurar a atividade física fora do período escolar, mas também evidenciam a importância dessa medida para se predizer o nível de atividade física da criança. No entanto, em nossos resultados foi encontrada uma boa reprodutibilidade na maioria das questões apresentadas, o que evidencia a possibilidade de utilização dessa estratégia para estudos que envolvam grande número de indivíduos na faixa etária de sete a dez anos de idade.

Embora evidências mais recentes destaquem a falta de concordância entre a atividade física relatada pelos pais comparada com aquela declarada pelos filhos adolescentes, ${ }^{16}$ nessa investigação os pais e cuidadores que responderam ao questionário eram responsáveis por crianças, onde o controle destes sobre as atividades praticadas pelos filhos pode ser maior, o que argumenta favoravelmente para os resultados encontrados.

A escolaridade das mães foi um pouco mais elevada, uma vez que em torno de $60 \%$ delas possuem mais de oito anos de estudo. Este fato pode ser um facilitador para o entendimento do instrumento e pode estar relacionado ao balanceamento do número de crianças advindas de escolas públicas e particulares. Este balanceamento, não observado no projeto Saúdes-Vitória, teve o objetivo de representar de modo semelhante diferentes níveis socioeconômicos e de escolaridade buscando avaliar a adequação de linguagem do questionário aos grupos estudados.

Os resultados das respostas do teste/re-teste, de um modo geral, sugerem uma boa reprodutibilidade do questionário em questão. Ressalta-se que a dispersão observada no relato do tempo dedicado à televisão, embora tenha apresentado coeficiente de reprodutibilidade moderado, revelou diferenças substanciais nas horas relatadas entre a primeira e segunda aplicação do questionário. Esse resultado possivelmente se atribui a inconstância do tempo dedicado a televisão.

Em recente publicação do Estudo Saúdes, devido à baixa frequência e baixa duração do uso de vídeo game, as informações sobre lazer sedentário, obtidas a partir a aplicação do presente questionário, foram agregadas considerando os itens de tempo dedicado à televisão, computador e videogame, em um indicador único de lazer sedentário. ${ }^{17}$

A comparação dos resultados desse estudo com outros realizados na população brasileira tornou-se difícil devido ao fato de existirem poucos estudos investigando a reprodutibilidade de questionários para a prática da atividade física nessa faixa etária.6,13 Em um estudo realizado em Florianópolis, ${ }^{6}$ no Estado de Santa Catarina, confirmou-se a consistência das informações obtidas com a aplicação no questionário DAFA. Contudo, o instrumento não avaliou a consistência das informações de caráter quantitativo avaliando o nível de atividade física (NAF), fato que limitou a possibilidade de classificar o NAF de acordo com o número de horas semanais.

O questionário Physical Activity Questionaire for Older Children (PAQ-C) ${ }^{18,19}$ tem sido empregado em estudos realizados em crianças, porém trata-se de um instrumento complexo para aplicação em indiví- 
duos muito jovens e requerem o auxílio dos pais para o seu preenchimento. 20

$\mathrm{O}$ instrumento utilizado nesta investigação se mostrou consistente para identificação da atividade física de deslocamento, lazer e modalidade esportiva, assim como para atividades sedentárias, especialmente o uso de computador, apresentando índices de concordância e correlação de moderados a altos, que são corroborados por outro estudo internacional. 11

Destaca-se que a prática do esporte 1 apresentou correlação moderada e pouca dispersão entre as réplicas, e que este resultado corrobora com outros estudos que verificaram maior reprodutibilidade na mensuração de atividades esportivas supostamente por serem mais sistematizadas. 21 No entanto,

\section{Referências}

1. Kesaniemi YK, Danforth EJr, Jensen MD, Kopelman PG, Lefèbvre P, Reeder BA. Dose-response issues concerning physical activity and health: an evidence-based symposium. Med Sci Sports Exerc. 2001; 33: 351-8.

2. Bauman AE. Updating the evidence that physical activity is good for health: an epidemiological review 2000-2003. J Sci Med Sport. 2004; 7: 6-19.

3. Alves JGB. Atividade física em crianças: promovendo a saúde do adulto. Rev Bras Saúde Matern Infant. 2003; 3: 5 6 [Editorial].

4. Lopes VP, Maia JAR. Actividade física nas crianças e jovens. Rev Bras Cineantropom Desempenho Hum. 2004; 6: 82-92.

5. Alves JGB, Montenegro FMU, Oliveira FA, Alves RV. Prática de esportes durante a adolescência e atividade física de lazer na vida adulta. Rev Bras Med Esporte. 2005; 11: 291-4.

6. Barros MVG, Assis MAA, Pires MC, Grossemann S, Vasconcelos FAG, Luna MEP, Barros SSH. Validity of physical activity and food consumption questionnaire for children aged seven to ten years old. Rev Bras Saúde Matern Infant. 2007; 7: 437-48.

7. Bastos JP, Araújo CLP, Hallal PC. Prevalence of insufficient physical activity and associated factors in brazilian adolescents. J Phys Act Health. 2008; 5: 777-94.

8. Dumith SC. Physical activity in Brazil: a systematic review. Cad Saúde Pública. 2009; 25 (Supl. 3): S415-26.

9. Lima RBCP. Padrão de atividade física em crianças e jovens: um breve resumo do estado do conhecimento. Rev Bras Cineantropom Desempenho Hum. 2000; 12: 68-76.

10. Bland M, Altman D. Measuring agreement in method comparison studies. Stat Methods Med Res. 1999; 8: 13560

11. Sirard JR, Pate RR. Physical activity assessment in children and adolescents. J Sports Med. 2001; 31: 439-54. contrariando as informações prévias, não se observou reprodutibilidade para a segunda modalidade de esporte.

À despeito das limitações apresentadas quanto a identificação do tempo de atividades físicas observou-se que o questionário empregado neste estudo parece ter boa reprodutibilidade, quando respondido pelas mães ou cuidadoras. No entanto há necessidade de estudos de validação, tendo em vista que uma das limitações dos métodos subjetivos para quantificar atividade física se pauta na variação de validade entre categorias de indivíduos. 22

Ressalta-se a importância deste questionário para investigação da atividade física, pois não foram identificados outros estudos nesta faixa etária, utilizando instrumentos quantitativos.

12. Welk GJ, Corbin CB, Dale D. Measure issues in the assessment of physical activity in children. Res Q Exerc Sport. 2000; 71 (Suppl. 2): S59-73.

13. Costa FF, Liparotti JR. Reprodutibilidade do questionário dia típico de atividade física e alimentação. Rev Bras Cineantropom Desempenho Hum. 2010; 12: 21-8.

14. Goodwin RA, Brulé D, Junkins EA, Dubois S, Beer-Borst $S$. Development of a food and activity record and a portionsize model booblet for use by 6- to 17- years olds: a review of focus-group testing. J Am Diet Assoc. 2001; 101: 926-8.

15. Vanhees L, Lefevre J, Philippaerts R, Martens M, Huygens W, Troosters T, Beunen G. How to assess physical activity? How to assess physical fitness? Eur J Cardiovasc Prev Rehabil. 2005; 12: 102-14.

16. Reichert FF, Menezes AMB, Araújo CL, Hallal PC. Selfreporting versus parental reporting of physical activity in adolescents: the 11-year follow-up of the 1993 Pelotas (Brazil) birth cohort study. Cad Saúde Pública. 2010; 26: 1921-7.

17. Molina MDCB, Faria CP, Montero MP, Cade NV, Mill JG Fatores de risco cardiovascular em crianças de 7 a 10 anos de área urbana, Vitória, Espírito Santo, Brasil. Cad Saúde Pública. 2010; 26: 909-17.

18. Silva MAM, Rivera IR, Ferraz MRMT, Pinheiro AJT, Alves SWS, Moura AA, Carvalho ACC. Prevalência de fatores de risco cardiovascular em crianças e adolescentes da rede de ensino da cidade de Maceio. Arq Bras Cardiol. 2005; 84: 387-92.

19. Rivera IR, Silva MA, Silva RD, Oliveira BA, Carvalho AC. Physical inactivity, TV-watching hours and body composition in children and adolescents. Arq Bras Cardiol. 2010; 95: 159-65.

20. Hodgkin E, Hamlin MJ, Ross JJ, Peters F. Obesity, energy intake and physical activity in rural and urban New Zealand children. Rural Remote Health. 2010; 10: 1336. Available from: http://www.rrh.org.au 
21. Guedes DP, Lopes CC, Guedes JERP, Stanganelli LC. Reprodutibilidade e validade do questionário Baecke para avaliação da atividade física habitual em adolescentes. Rev Port Cien Desp. 2006; 6: 265-74.

22. Warren JM, Ekelund U, Besson H, Mezzani A, Geladas N, Vanhees L. Experts panel assessment of physical activity - a review of methodologies with reference to epidemiological research: a report of the exercise physiology section of the European Association of Cardiovascular Prevention and Rehabilitation. Eur J Cardiovasc Prev Rehabil. 2010; 7: 127-39.

Recebido em 29 de junho de 2010

Versão final apresentada em 26 de abril de 2011

Aprovado em 2 de maio de 2011 